\title{
Building a megabrain atlas
}

A digital platform has been created to incorporate multiple brain data resources into a common global mouse atlas.

Navigating the brain's complexity can be a daunting task. Digital atlases help neuroscientists disentangle some of the brain's spatial heterogeneity, but these atlases are often focused on one particular aspect—be it the representation of the different anatomical structures in the brain or the specific gene or protein expression patterns. As more of these resources become available, the need arises to connect data from these multiple and remote sources and integrate them into a common, more comprehensive global map.

The International Neuroinformatics Coordinating Facility (INCF) is a professional organization whose goal is to further the understanding of the brain by providing neuroscientists with a framework to share and integrate data. Among its initiatives, the INCF has launched the Digital Atlasing proj- ect, an international effort to create a publicly available atlas framework of the mouse brain that can link different types of databases within a common digital platform.

To build this resource, an INCF Digital Atlasing task force established a standardized spatial coordinate system and built a canonical atlas space called the Waxholm space (WHS; http://waxholm.incf.org/). WHS was developed using high-resolution magnetic resonance imaging and matched histological series data of a single male adult C57Bl/6J brain specimen. This provides a three-dimensional reference atlas into which existing or future mouse data resources can be easily incorporated once they are registered in the WHS coordinate system.

A first prototype of INCF's Digital Atlasing project has recently been launched that integrates three major community resources into the WHS developing framework as atlas hubs: the Allen brain reference atlas, which is a database of over 20,000 in situ gene expression patterns of the adult mouse brain; the Edinburgh mouse atlas project, which is a digital atlas of mouse development; and the whole brain catalog, a multiscale virtual catalog of the mouse brain. The Digital Atlasing task force has registered the atlases to WHS, and the data are accessible via the atlas' own websites and associated web services.

The INCF Digital Atlasing project provides continuous supporting infrastructure to facilitate data access and exchange, and its members will be conducting workshops to help people register their data to the WHS and become part of this global endeavor. A collaborative effort from the wide neuroscience community will be essential to give the project richness akin to that of the brain.

\section{Erika Pastrana}

RESEARCH PAPERS

Hawrylycz, M. et al. Digital Atlasing and standardization in the mouse brain. PLoS Comput. Biol. 7, e1001065 (2011). 